\title{
Extended Atmospheres of the M Supergiants Alpha Ori and Alpha Sco
}

\author{
Alexander Brown and Graham M. Harper \\ Center for Astrophysics and Space Astronomy, University of Colorado, \\ Boulder, CO 80309-0389, USA
}

\begin{abstract}
Multifrequency spatially-resolved radio continuum observations of the M supergiants, $\alpha$ Ori (M2 Iab) and $\alpha$ Sco (M1.5 Iab + B2.5 V), have been obtained using the VLA A array and VLA+Pie Town configurations, to study changes in the extended (i.e. scale 1 - 10 stellar radii) atmospheres of these stars and to model the conditions in their wind acceleration regions. Strong modelling constraints on the atmospheric thermal properties are derived, because the radio emission is resolved at multiple wavelengths. Changes seen in the $\alpha$ Ori source flux density and radio visibility data occurring on several year timescales are described, based on observations obtained in 2002 February and April and in 1996 December. The need for multicomponent models of the plasma conditions in both the warm and cool gas around $\alpha$ Ori is discussed. The radio properties of the $\alpha$ Sco system, both of the M supergiant itself and in the $\mathrm{H}$ II region surrounding the B-type companion, provide important tools for estimating conditions within the $\mathrm{M}$ supergiant's wind.
\end{abstract}

\section{Introduction}

Thermal radio continuum emission is a powerful diagnostic of conditions within the extended atmospheres and winds of cool $\mathrm{K}$ and $\mathrm{M}$ giants and supergiants. Radio continuum data play a crucial role in determining the mass loss properties of evolved late-type stars because they directly sample conditions within the wind, yet their only sensitivity to the velocity field comes through the density structure implied by the continuity equation. Accurate mass loss rates and the processes that cause mass loss from most evolved cool stars are still poorly known but are of considerable importance for understanding stellar evolution.

The nearest $M$ supergiants have photospheric angular diameters of $\sim 50$ milliarcseconds (e.g. $11 \mu \mathrm{m}$ angular diameters of $56.6 \pm 1$ and $44.4 \pm 1$ mas, measured by Bester et al. (1996) for $\alpha$ Ori and $\alpha$ Sco, respectively). The largest array configuration ("A array") of the NRAO Very Large Array (VLA) can resolve radio sources with sizes of this order, particularly if the signal from the VLBA Pie Town antenna is correlated as part of the VLA. Use of the Pie Town antenna doubles the length of the available baselines. If a thermal radio 


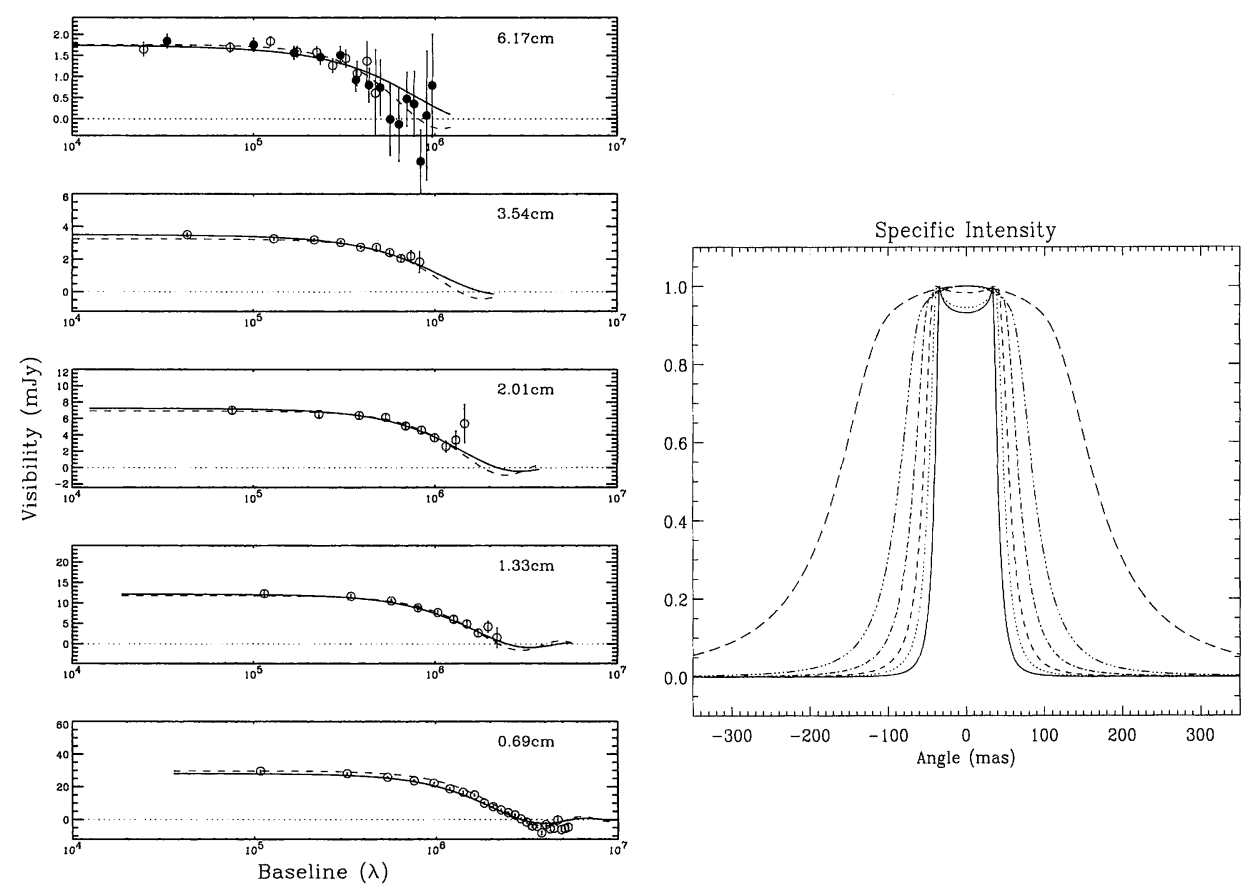

Figure 1. (left) Observed visibilities as a function of baseline for $\alpha$ Ori using VLA A array data only; (right) specific intensity distributions calculated from the Harper, Brown, \& Lim 2001 model showing predicted angular extent of the $\alpha$ Ori radio emission at wavelengths of 0.7 (smallest), 1.3, 2, 3.5, 6, and 20 (largest) $\mathrm{cm}$.

source can be resolved at different frequencies, a detailed model of the mean temperature structure can be derived.

Previous VLA observations of Betelgeuse ( $\alpha$ Ori) resolved or partially resolved the stellar atmosphere at multiple wavelengths (e.g. Lim et al. 1998). Our fits to the Lim data (see Fig. 1 left) show that $\alpha$ Ori is fully (i.e. beyond the first null) or significantly resolved in all VLA observing bands. These observations allowed us to establish the first detailed semi-empirical mean model of a spatially extended atmosphere covering the region from $1<R\left(R_{*}\right)<10$ (Harper, Brown \& Lim, 2001); the key region for studying the wind acceleration. One of the initial surprises from analysis of the radio visibility data is that the mean atmosphere is quite cool with $1500<T_{e}(K)<4000$; in contradiction to previous semi-empirical and theoretical estimates of $T_{e} \sim 8000 \mathrm{~K}$. Because the radio emission is thermal and on the Rayleigh-Jeans tail $\left(S_{\nu} \sim T_{e}\right)$, the mean semi-empirical model is not heavily biased by small components of hot plasma, unlike the UV where small amounts of hot plasma can dominate the total stellar emission. The resolution of the apparent difference between earlier thinking and the direct observation of cool extended atmospheres is simple: without spatial information many possible models were compatible with the observed radio 
fluxes, ranging from cool and extended to warm and compact. The atmosphere of $\alpha$ Ori is thermally-complex with a multi-component structure containing both hot (UV emission, $\mathrm{H} \alpha$ scattering) and cold (radio emission) structures in close juxtaposition. The hot $(8000 \mathrm{~K})$ plasma has a filling factor of less than $25 \%$. High resolution UV spectroscopy of Betelgeuse has shown that the theoretical Alfvén wave driven wind model of Hartmann \& Avrett (1984) is not a good description of the hot ionized plasma.

In their pioneering study on Antares ( $\alpha$ Sco), Hjellming \& Newell (1983), using VLA data obtained in 1982-3, found that the M1.5 Iab primary of $\alpha$ Sco had similar radio properties to $\alpha$ Ori (M2 Iab), and that the B2.5 V companion created an optically thin ionized cavity in the supergiant wind. They derived a simple model which provided a good match to the observed ionization cavity, whose shape is an excellent diagnostic for the mass-loss rate of the M star.

\section{Observations}

We have obtained new observations of $\alpha$ Sco using the VLA A array (36 km max. baseline) on 1998 May 29 at 6, 3.5, 2, 1.3 and $0.7 \mathrm{~cm}$, and of $\alpha$ Ori using the A array + Pie Town Link on 2002 February 17 and April 12 at 20, 6, 3.5, 2 , and $1.3 \mathrm{~cm}$. Fast switching using a close phase calibrator (either $0532+075$ or 1626-298) was used at $2,1.3$, and $0.7 \mathrm{~cm}$ to monitor the tropospheric and ionospheric phase drifts on 2-3 minute timescales. These continuum data were calibrated using standard AIPS procedures and were analysed using both AIPS and our own customized IDL visibility analysis routines.

\section{Data Analysis and Modelling}

The radio emission from $\alpha$ Ori and $\alpha$ Sco is well resolved in our observations with source sizes several times larger than the photospheric diameter. Different frequencies sample different portions of the extended atmosphere - higher frequencies forming closer to the stellar surface. The contributions functions as a function of radius derived from our (Harper, Brown, \& Lim) model of $\alpha$ Ori show that the $20 \mathrm{~cm}$ emission originates near $4 \mathrm{R}_{*}$, while $2 \mathrm{~cm}$ emission peaks at 1.4 $\mathrm{R}_{*}$. The distribution of the radio emission on the sky can also be calculated from our models and the predicted specific intensities are illustrated in Fig. 1.

We model the complex visibility distribution to determine the source morphology. We have developed state-of-the-art wind/atmosphere models, that constrain the density and temperature structure, and thus determine the mass loss rate and wind velocity law. Our models follow a unified approach to the atmospheres of late-type stars, using both radio and ultraviolet data to model the chromospheres and winds of evolved cool stars in a self-consistent way. Modelling the radio emission requires careful treatment of ionization. Our technique uses spherical geometry and escape probability techniques with a detailed hydrogen ionization model including advection terms in the rate equations. The inclusion of advection and the consequent "freezing in" of ionization leads to significantly higher ionization in the cool expanding wind and, hence greater radio emission for the same wind parameters. The winds are often only partially 


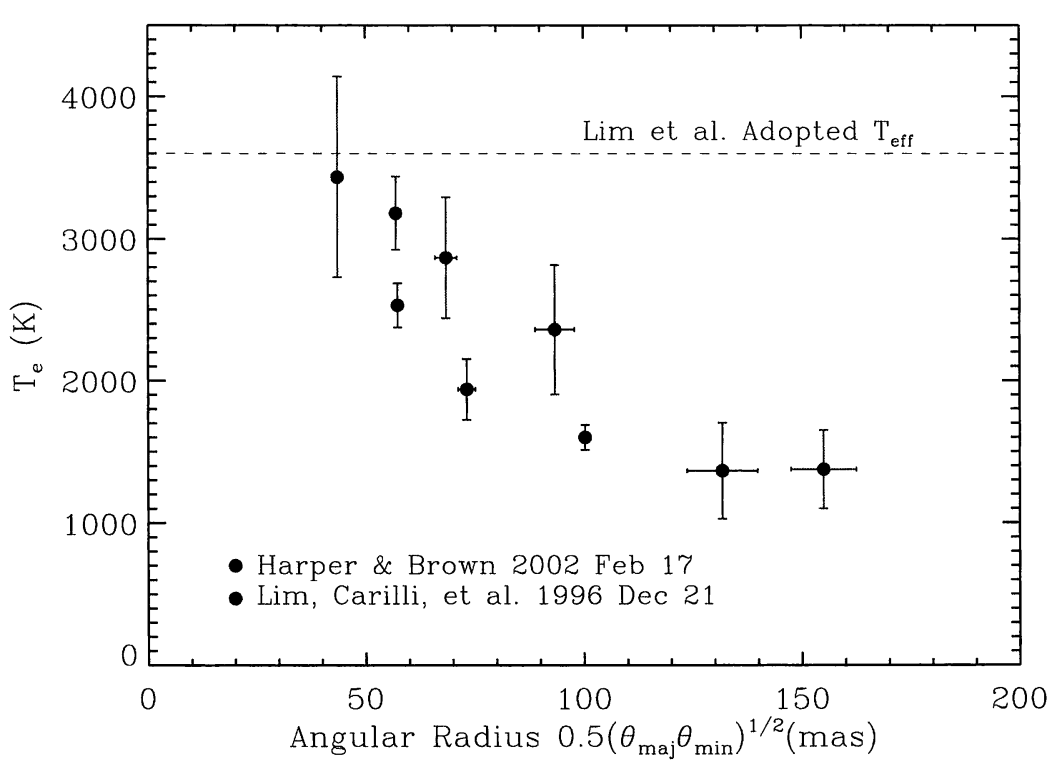

Figure 2. Variable temperature structure of the wind of $\alpha$ Ori. The upper red symbols are the values derived from the 1996 data, while the blue symbols show our 2002 measurements of source size and temperature.

ionized and the emission is from the region, critical to studying the physics of the mass loss process, where the wind is still accelerating.

Our 2002 data show that the radio emission from $\alpha$ Ori has faded over the six years since the Lim data were obtained in 1996 December (see Fig. 2). However, the source sizes are comparable, and the flux density reduction seems to be related to a decrease in mean atmospheric temperature. Fitting a uniform 2-D ellipse - a good intensity model based on the predicted specific intensities to $\mathrm{K}$ band $(1.3 \mathrm{~cm}) 2002$ February complex visibilities leads to major and minor axes of 117 and 113 mas, which differ by only $3 \%$. The $\mathrm{X}$ band $(3.5 \mathrm{~cm})$ data show a more asymmetric source with a major axis $30 \%$ larger than the minor axis. Only very small changes in flux density are seen between 2002 February and April.

Our new, more extensive A array observations of $\alpha$ Sco and detailed 3-D modelling of the radio emission confirm the basic results of Hjellming \& Newell (1983). The emission from the $\mathrm{M}$ supergiant is well resolved in multiple observing bands: a 2-D uniform ellipse fit to the $\mathrm{K}$ band $(1.3 \mathrm{~cm})$ complex visibilities estimates major and minor axes of $163 \pm 20$ and $107 \pm 9$ mas. Thus, the emission is highly asymmetric and $\alpha$ Sco is actually larger than $\alpha$ Ori at 1.3 $\mathrm{cm}$. The brightness temperatures are similar to those of $\alpha$ Ori. Our model prediction of the $\mathrm{X}$ band brightness distribution is in remarkable agreement with the observations, particularly with regard to the location and size of the emission around the B star. This confirms that this emission is due to the 
ionized gas within the H II region. Our H II region flux densities agree well with those of Hjellming \& Newell, which is to be expected because the H II region is controlled by the constant B star photospheric Lyman continuum flux.

\section{Summary of Results}

The radio continuum emission associated with the atmospheres of $\alpha$ Ori and $\alpha$ Sco is significantly (several stellar radii) extended and resolved by VLA A array and A array + Pie Town observations; thereby allowing detailed modelling of the inner wind structure. The winds from M supergiants, like $\alpha$ Ori and $\alpha$ Sco, are cooler (maximum temperature less than $4000 \mathrm{~K}$, falling to below $1000 \mathrm{~K}$ beyond $\left.9 \mathrm{R}_{*}\right)$ than previously thought. Hot $(8000 \mathrm{~K})$ plasma is also present embedded within the cooler wind. The atmosphere of $\alpha$ Ori is cool enough that $\mathrm{H}^{-}$free-free emission is the dominant radio emission process. Fitting the complex visibility distributions suggests that the radio sources can be significantly asymmetric. The brightness of the radio emission from both $\alpha$ Ori and $\alpha$ Sco varies significantly on several year timescales. $\alpha$ Sco shows resolved emission from both the $\mathrm{M}$ supergiant atmosphere and from an H II region surrounding the B dwarf secondary.

Acknowledgments. This work is supported NSF grant AST-0206367 and NASA grants NAG5-4804 and NAG5-13058 to the University of Colorado. The VLA is operated by NRAO, whose staff we thank for the assistance - the National Radio Astronomy Observatory is a facility of the National Science Foundation operated under cooperative agreement by Associated Universities, Inc.

\section{References}

Bester, M., et al. 1996, AJ, 463, 336

Harper, G.M., Brown, A., \& Lim, J. 2001, ApJ, 551, 1073

Hartmann, L., \& Avrett, E.H. 1984, ApJ, 284, 238

Hjellming, R.M., \& Newell, R.T. 1983, ApJ, 275, 704

Lim, J., et al. 1998, Nature, 392, 575 